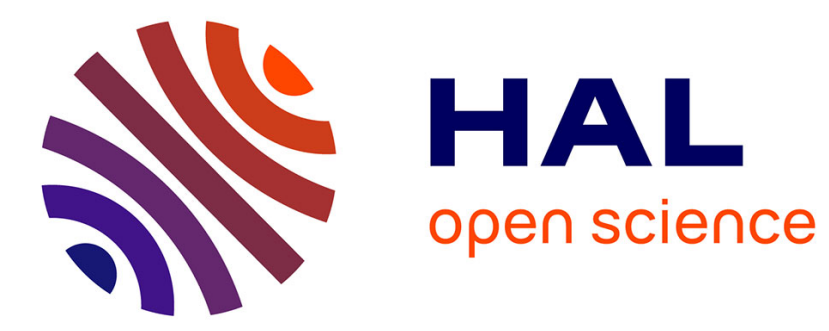

\title{
The Microstructural Effects of Randomly Generated Porous Media on Interfacial Convective Heat Transfer
}

Eren Ucar, Moghtada Mobedi, Azita Ahmadi-Senichault

\section{To cite this version:}

Eren Ucar, Moghtada Mobedi, Azita Ahmadi-Senichault. The Microstructural Effects of Randomly Generated Porous Media on Interfacial Convective Heat Transfer. Int. Symp. on Convective Heat and Mass Transfer, Jun 2014, Kusadasi, Turkey. 12p. hal-01174019

\author{
HAL Id: hal-01174019 \\ https://hal.science/hal-01174019
}

Submitted on 8 Sep 2015

HAL is a multi-disciplinary open access archive for the deposit and dissemination of scientific research documents, whether they are published or not. The documents may come from teaching and research institutions in France or abroad, or from public or private research centers.
L'archive ouverte pluridisciplinaire HAL, est destinée au dépôt et à la diffusion de documents scientifiques de niveau recherche, publiés ou non, émanant des établissements d'enseignement et de recherche français ou étrangers, des laboratoires publics ou privés. 


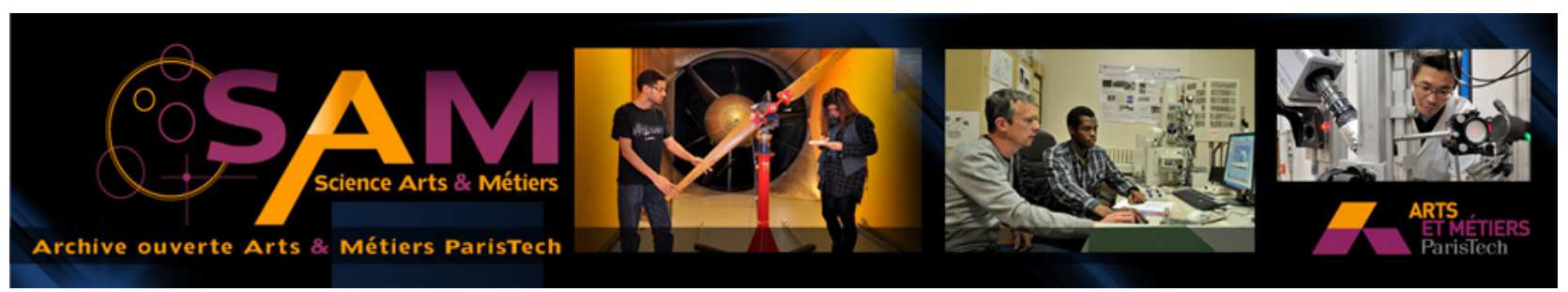

\section{Science Arts \& Métiers (SAM)}

is an open access repository that collects the work of Arts et Métiers ParisTech researchers and makes it freely available over the web where possible.

This is an author-deposited version published in: http://sam.ensam.eu

Handle ID: .http://hdl.handle.net/10985/9721

\section{To cite this version :}

Eren UCAR, Moghtada MOBEDI, Azita AHMADI-SENICHAULT - The Microstructural Effects of Randomly Generated Porous Media on Interfacial Convective Heat Transfer - In: Int. Symp. on Convective Heat and Mass Transfer, Turquie, 2014-06 - Proceedings of CONV-14: Int. Symp. on Convective Heat and Mass Transfer - 2014 


\title{
THE MICROSTRUCTURAL EFFECTS OF RANDOMLY GENERATED POROUS MEDIA ON INTERFACIAL CONVECTIVE HEAT TRANSFER
}

\author{
Eren Ucar $^{1}$ Moghtada Mobedi ${ }^{1, *}$ and Azita Ahmadi ${ }^{2}$ \\ ${ }^{1}$ Mechanical Engineering Department, Izmir Institute of Technology, Urla 35430, Izmir, Turkey \\ ${ }^{2}$ I2M - TREFLE Department, UMR CNRS 5295, Arts et Metiers ParisTech, Esplanade des Arts et \\ Metiers, 33405 Talence Cedex, France \\ (* Corresponding author: moghtadamobedi@iyte.edu.tr)
}

\begin{abstract}
Heat and momentum transfer in a random generated porous media is investigated. 6 different random porous media are generated using a Monte-Carlo (MC) procedure. The continuity, momentum and energy equations are solved for a row of cylinders with a square cross-section (i.e. rods) representing the entire domain of a random porous medium. The microstructure properties of the random porous media such as the mean nearest neighbor distance and the standard deviations of Voronoi areas, nearest neighbor distance and neighbor orientation for each generated random porous medium are obtained and compared with each other. The velocity and temperature fields in the porous media are shown via isotherms and streamlines. The rods in the domain are classified into three groups as blocker, active and passive rods based on our observations from the temperature field and the variations of the Nusselt number of each rod. The preliminary obtained results show that it may be possible to predict the interfacial heat transfer coefficient in a random porous medium based on some microstructure properties.
\end{abstract}

\section{INTRODUCTION}

Coupled heat transfer and fluid flow through porous media occurs in nature and many industrial applications. The flow of air through a lung, water flow in soil, fluid flow through metal or ceramic foams, drying processes in food industry are some examples involving heat and fluid flow through porous media. Microscopic or macroscopic approaches can be used to simulate heat and fluid flow in a porous medium. The macroscopic approach is widely used by many researchers and employed in many commercial codes. In order to perform a macroscopic analysis of heat transfer and flow in a porous medium, macroscopic properties such as permeability, interfacial heat transfer coefficient or thermal dispersion coefficient should be known. These macroscopic properties can be found experimentally. However, recent developments in computational methods facilitate the determination of these coefficient numerically. The velocity, pressure and temperature fields can be calculated at the microscale level and then macroscopic properties can be found from the obtained computational results.

Several numerical studies related to the analysis of heat and fluid flow in a porous medium at the microscopic scale can be found in the literature. Nakayama et al. [2002] carried out a numerical study of heat and fluid flow in a two dimensional, periodic and anisotropic porous medium consisting of square rods. The effects of the vertical distance between the rods, the macroscopic flow angles and Reynolds number on the interfacial heat transfer coefficient were analyzed. Convective heat transfers in two dimensional periodic porous structures involving square bars were also analyzed by Lopez Penha et al. [2012]. It was shown that the interfacial Nusselt number does not change significantly for high ratios of solid-to-fluid thermal conductivity while it changes for low ratios. Another study on heat and fluid flow in periodic porous structures was conducted by Gamrat et al. [2008]. Correlations for the 
determination of interfacial Nusselt number as a function of porosity, Reynolds number and Prandtl number were proposed.

Literature survey shows that the number of microscopic scale analyses of fluid flow in randomly generated porous media is less than those for periodic structures. Microscale fluid flow in different random arrays of cylindrical fibers were analyzed by Yazdchi et al. [2012]. The study indicated that there is a relationship between permeability and the nearest neighbor distances of fibers. Chen and Papathanasiouming [2007-2008] developed a model for permeability of randomly generated porous structures consisting of cylindrical fibers. A relation for determination of permeability based on the mean nearest inter-fiber spacing was proposed. Another pore level simulation for randomly generated porous media consisting of rectangular rods was carried out by Nabovati and Sousa [2007]. The study showed that the permeability of random porous media is lower than the permeability of regularly ordered media. Moreover, a correlation between the average tortuosity and the porosity of the considered porous medium was proposed in this study. A numerical study on heat and fluid flow for randomly distributed porous media was done by Rahimian et al. [2002]. The porous medium was two dimensional with square rods. The average and local Nusselt numbers were calculated and compared with the empirical data in this study. Non uniform flow and heat transfer due to the distribution of void fractions in random packed bed was discussed and analyzed by Guo and Dai [2010]. The analyses were made for Reynolds numbers between 4.6 - 56.2 which characteristic length was defined based on sphere diameters. The temperature field and wall effects were investigated in details. It was observed that the flow inhomogeneity increases with increasing inflow Reynolds number and higher temperature gradients were viewed for low void fractions.

The aim of the present study is to investigate the effects of microstructural parameters on heat and fluid flow in randomly generated porous media consisting of square bars. Firstly, the random porous media are generated and their microstructure properties such as Voronoi area, nearest neighbor distance and neighbor orientation standard deviations (i.e., SD) and mean nearest neighbor distance are obtained and presented. The continuity, momentum and energy equations are solved for the randomly generated porous media and the velocity, pressure and temperature fields are obtained. These fields are shown via streamlines and isotherms contours. The interfacial heat transfer coefficients of different generated porous media are computed, numerically. Heat and fluid flow in periodic and random structures are compared. Rods are classified according to Nusselt number of each rod and their effects on heat and fluid flow. The obtained results show that overall Nusselt number of randomly generated porous media is generally greater than regular porous media composed of periodic inline arrangement of rods. However, a case for which the overall Nusselt numbers of random and inline arrangements are almost equal is observed. The present study shows that it is possible to derive a relation between interfacial convective heat transfer coefficients and microstructure properties of a randomly generated porous medium.

\section{PHYSICAL MODEL}

The physical model considered in the present study is shown in Figure 1. A randomly generated two dimensional porous domain consisting of long solid bars is investigated in this study. In order to reduce the size of the computational domain and increase the length of the porous medium in the flow direction (i.e., $x$ direction), the structure of the porous medium in the y direction is periodic; while the arrangement of bars in the $\mathrm{x}$ direction is completely random, as seen from Figure 1. Hence, for our computational study, a Representative Elementary Volume (REV) which is a row of the considered porous medium is taken into consideration. The height of the REV is considered as $\mathrm{H}$ while the length is $14 \mathrm{H}$ with a clear region without any bars at the outlet to apply the outlet boundary condition which is negligible gradients of all dependent variables in the flow direction. 
The solid rods have a square cross-section with an edge of length $h$. In this study, the results are obtained for the porosity value of 0.75 and the edge of the solid rods is equal to the half of the height of the REV $(h=H / 2)$. The top and bottom of the REV are symmetrical and the inlet velocity and temperature distributions are uniform. The flow is laminar and the study is performed for air. The temperature of all solid rods is maintained at $\mathrm{T}_{\mathrm{w}}$ which is higher than inlet temperature (i.e., $\mathrm{T}_{\mathrm{i}}$ ). The number of rods is sufficiently large to observe periodic heat and fluid flow through flow direction even at low Reynolds number.

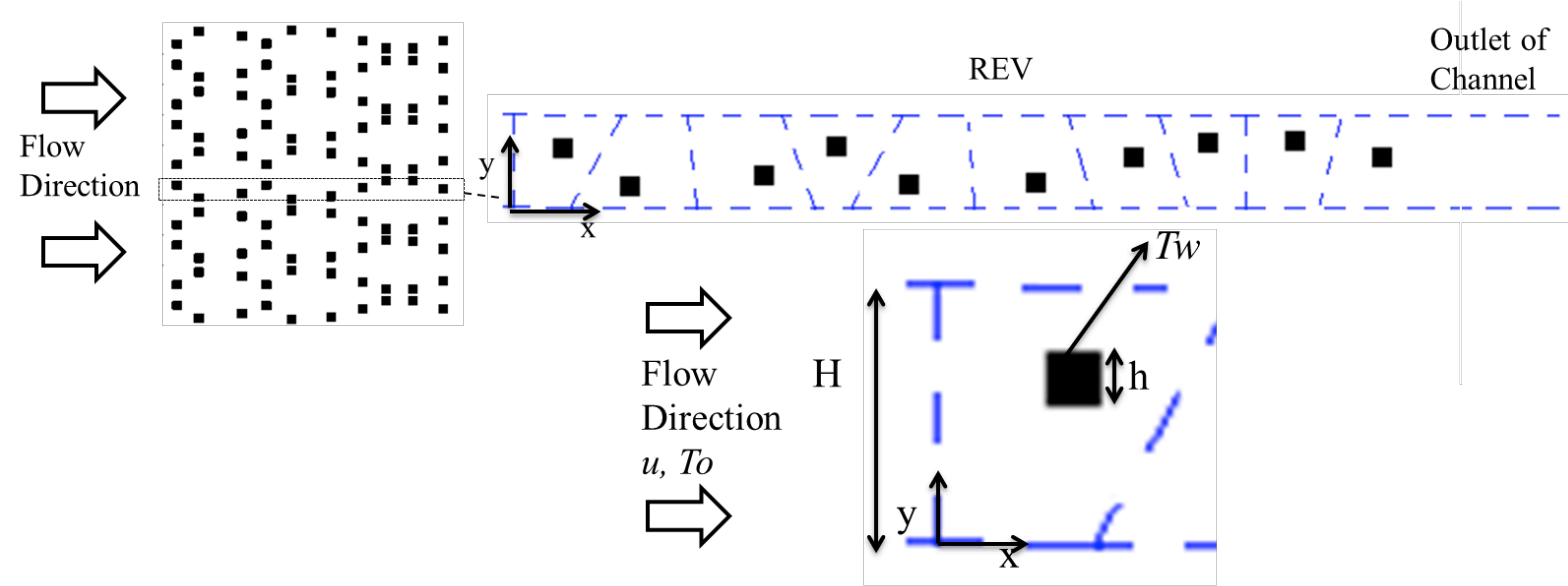

Figure 1. The studied random porous media and Representative Elementary Volume.

\section{METHOD OF GENERATION OF MICROSTRUCTURE IN THE CONSIDERED PHYSICAL MODEL}

The 'random' rods distribution for the studied REV is generated by using Monte-Carlo (MC) procedure. Firstly, the centre locations of the square rods for a periodic, isotropic and regular porous structure with inline arrangement are generated. Then, the centre location of each rod is perturbed in a random direction with a random displacement. The maximum random displacement is fixed for all rods. This means that the perturbation of each rod is limited so that it cannot enter the territory of the neighbouring rods. One round of perturbation is completed when all square rods are perturbed and have new centres. The perturbation continues until the number of perturbation round attains the desired value. It should be mentioned that porosity, number of square rods, maximum allowable distance for rod center and number of perturbation rounds should be known before the generation of random distributions. In this study, the values of porosity, allowable distance, and number of perturbation are considered as $0.75,0.25$ and $10^{6}$, respectively.

\section{GOVERNING EQUATIONS, BOUNDARY CONDITIONS AND SOLUTION METHODS}

The flow is two dimensional, steady and laminar while the fluid is incompressible and Newtonian. The thermo-physical properties of the fluid are assumed to be constant. The governing equations for the fluid flow are continuity and momentum equations which can be written in the following form:

$$
\begin{gathered}
\frac{\partial \mathrm{u}}{\partial x}+\frac{\partial \mathrm{v}}{\partial y}=0 \\
\mathrm{u} \frac{\partial \mathrm{u}}{\partial x}+\mathrm{v} \frac{\partial \mathrm{u}}{\partial y}=-\frac{1}{\rho} \frac{\partial P}{\partial x}+v\left(\frac{\partial^{2} \mathrm{u}}{\partial x^{2}}+\frac{\partial^{2} \mathrm{u}}{\partial y^{2}}\right)
\end{gathered}
$$




$$
\mathrm{u} \frac{\partial \mathrm{v}}{\partial x}+\mathrm{v} \frac{\partial \mathrm{v}}{\partial y}=-\frac{1}{\rho} \frac{\partial P}{\partial y}+v\left(\frac{\partial^{2} \mathrm{v}}{\partial x^{2}}+\frac{\partial^{2} \mathrm{v}}{\partial y^{2}}\right)
$$

where $u$ and $v$ are velocity components in $x$ and $y$ directions, and $p$ represents pressure. Equations 13 are solved for the fluid flow in the voids between the solid bars in order to determine the velocity and pressure distributions. Then, by using the obtained velocity field, the steady energy equation (Eq. 4) is solved to obtain fluid temperature distributions in the voids.

$$
\mathrm{u} \frac{\partial T_{f}}{\partial x}+\mathrm{v} \frac{\partial T_{f}}{\partial y}=\alpha_{f}\left(\frac{\partial^{2} T_{f}}{\partial x^{2}}+\frac{\partial^{2} T_{f}}{\partial y^{2}}\right)
$$

where $\alpha_{\mathrm{f}}$ is the thermal diffusivity of the fluid. Boundary conditions for the considered REV can be written as follows;

On solid walls,

$$
\mathrm{u}=\mathrm{v}=0, T_{f}=T_{s}
$$

For top and bottom boundaries, $\quad \frac{\partial \mathrm{u}}{\partial y}=\frac{\partial \mathrm{v}}{\partial y}=\frac{\partial T_{f}}{\partial y}=0$

For inlet boundary condition,

$$
\mathrm{u}=\mathrm{u}_{i}, T=T_{i}
$$

For outlet boundary condition, $\quad \frac{\partial \mathrm{u}}{\partial x}=\frac{\partial \mathrm{v}}{\partial x}=\frac{\partial T_{f}}{\partial x}=0$

where $u_{i}$ and $T_{i}$ are inlet velocity and temperature, and $T_{s}$ is the surface temperature. The heat flux at any point between the solid and fluid phases at the interface can be calculated by using Fourier law due to no slip boundary condition. The integral of heat flux over the total interface area yields heat transfer rate. Then, the interfacial heat transfer coefficient can be determined as follows:

$$
h_{s f} A_{s s}\left(T_{s}-\left\langle T_{f}\right\rangle^{f}\right)=\frac{1}{V} \int_{A_{s f}} \vec{n} \cdot k_{f} \vec{\nabla} T_{f} d A
$$

where $h_{s f}$ is the interfacial convective heat transfer coefficient, $\mathrm{k}_{\mathrm{f}}$ is the thermal conductivity of fluid and $A_{S S}$ the specific solid-fluid interface area which is the total solid-fluid interface area per unit volume of a porous medium. In this study, the interfacial convective heat transfer coefficient for each cell in the flow direction is calculated based on the difference between the bar surface temperature and the volume average of fluid temperature in its Voronoi polygon. Therefore, the intrinsic volume average of the fluid region $\left\langle\mathrm{T}_{\mathrm{f}}>^{\mathrm{f}}\right.$ is calculated by using Voronoi area of each solid bar. The interfacial Nusselt number for each rod can be defined as follows:

$$
\bar{N} u_{\text {rod }}=\frac{h_{s f} H}{k_{f}}
$$

where $\mathrm{H}$ is the height of REV. The average Nusselt number of the porous media is the arithmetic average of the Nusselt number of the rods since the size of all rods are equal. 


$$
\bar{N} u_{\text {overall }}=\frac{\sum_{i=1}^{n} \bar{N} u_{\text {rod }}}{n}
$$

The above governing equations are solved using a commercial software based on finite volumes. The power law scheme is used to treat the discretization of convection terms (i.e., inertia terms) in Eqs. (2) and (3).Based on our numerical experience, the over-relaxation values for continuity, momentum and energy equations are $0.3,0.7$ and 1 respectively. The residual convergence for flow equations are $10^{-9}$ for continuity, momentum and energy equations. Our observation showed that it will be sufficient to discretize the channel height to 100 whereas the channel length is divided into 1400 providing square meshes for the computational domain.

\section{CHARACTERIZATION OF MICROSTRUCTURE PROPERTIES}

In order to distinguish different random porous media from each other and to predict the important characteristic parameters which affect heat and fluid flow through the random porous media, some microstructural statistical descriptors are required. Literature survey shows that several statistical and structural descriptors can be used to characterize a random porous medium.

\section{Voronoi Tessellation and standard deviation of Voronoi areas}

A plane involving many points can be divided into sub-regions so that each point has a territory which is closer to that point than to other points. The combination of the territories results in a pattern of packed convex polygons covering the whole plane. This constructed pattern is known as the Dirichlet tessellation of the points and the sub-regions called as Voronoi cells [Bowyer, 1981]. A periodic and isotropic porous medium with inline arrangement may have Voronoi cell polygons with 4 sides and vertex angles of $90^{\circ}$ while irregular Voronoi polygons are obtained for a nonisotropic and non-periodical porous medium as shown in Figure 2.

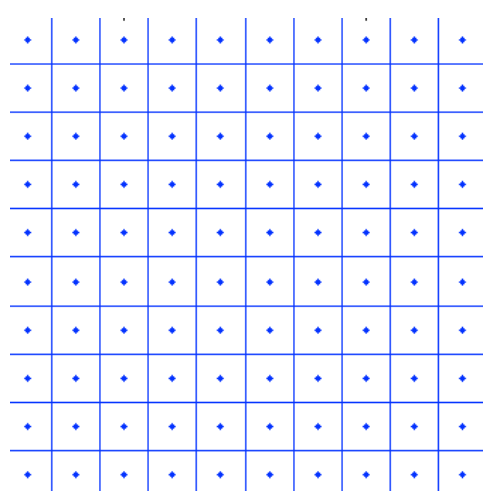

(a)

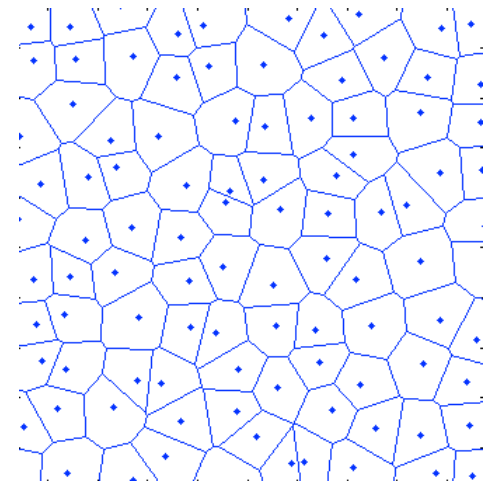

(b)

Figure 2. Voronoi polygons for a plane containing 100 points a) periodic and isotropic distributed points b) irregular and non-periodic distributed points

Voronoi polygon areas of the studied domain may be used to have an idea on the distribution of the solid rods. The standard deviation of the areas of the Voronoi polygons can also be used to determine the periodicity level of a porous medium. In a periodic porous medium, all Voronoi polygon areas are equal; hence the standard deviation of the areas is zero. When the distribution of the rods diverges from a periodic structure, the standard deviation will be greater than zero. 


\section{Nearest Neighbour Concept}

When a side of a Voronoi polygon is shared by the territory of two rods, these rods are neighbors. The neighbor distances are center to center distances between neighboring rods. Figure 3 shows the distribution of the nearest neighbors of a randomly generated porous medium. The red lines indicated the Voronoi polygons while the black lines show the nearest neighbors for each rod. As it can be predicted from the Figure 3, the distribution of neighbor distances of different randomly generated porous media is different. Therefore, the distribution of the nearest neighboring can be used as microstructure properties of a random porous media.

The standard deviation of the nearest neighbor distance of a periodic and isotropic porous medium with inline arrangement should be zero because of the fact that the distances between all square rods are identical. However, the nearest neighbor distance changes with the irregularity. Hence, the standard deviation of the nearest neighbor distance can also be used to specify a random porous medium. Moreover, the nearest neighbor orientation, which is the angle of nearest neighbor line with horizontal axis in clockwise direction, can also be used as a microstructural parameter for specification of a random porous media. The average of the nearest neighbor distance of the entire domain can also be used as a characteristic descriptor [Chen and Papathanasiouming 2007].

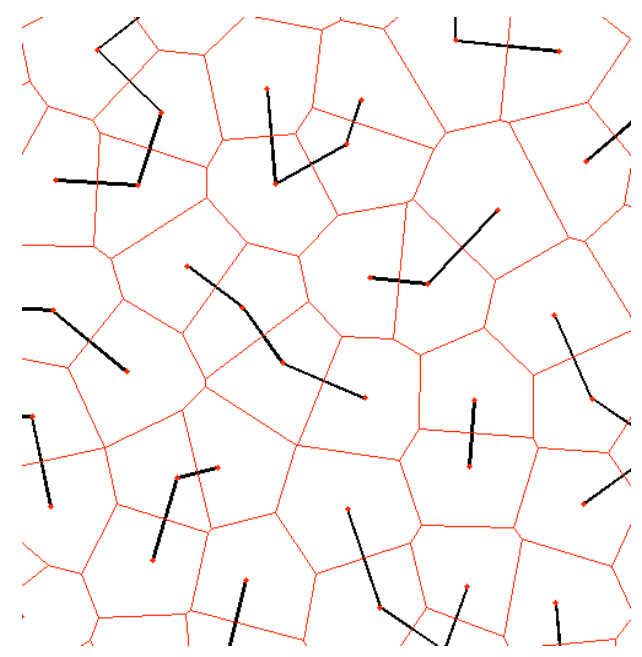

Figure 3. Nearest Neighbors in a random domain

\section{RESULT AND DISCUSSION}

In order to verify the obtained results, the interfacial Nusselt number for a periodic porous medium consisting of square rods with inline arrangement is obtained and compared with the available values in the literature (refs). The Figure 4 shows the comparison of the interfacial Nusselt number of the present study with the reported interfacial Nusselt numbers for a periodic porous medium consisting of an inline arrangement of square rods with a porosity of 0.75 . As can be seen, a good agreement exits between our results and the reported ones which validates the correctness of the present study.

\section{The microstructure properties of generated random porous media}

In this study, a periodic and 6 different random porous media consisting of square rods with a porosity value of 0.75 are studied. The Voronoi polygons along with the nearest neighbor 
distributions for a sample of the studied random porous medium are shown in Figure 5. The blue lines around the black square rods indicate Voronoi polygons while the red lines between centers of the square rods indicate nearest neighbors' distances for each rod.

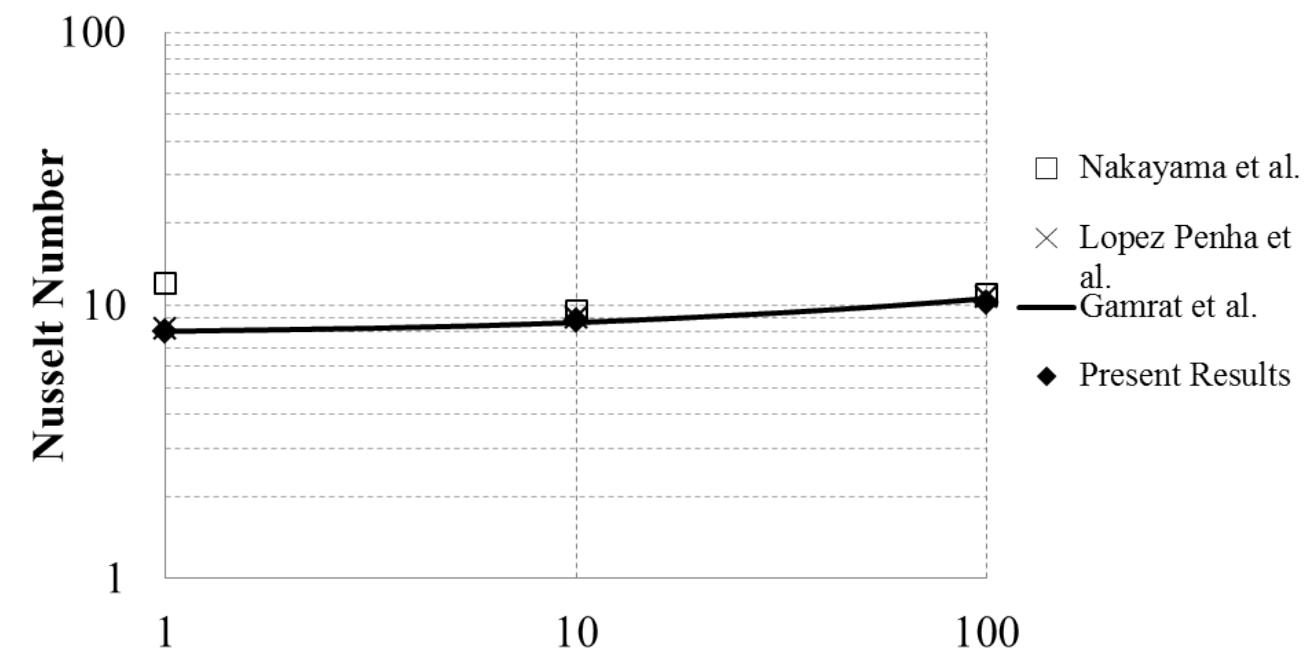

Reynolds Number

Figure 4. The comparison of interfacial Nusselt number obtained by the present study with the results reported in the literature.

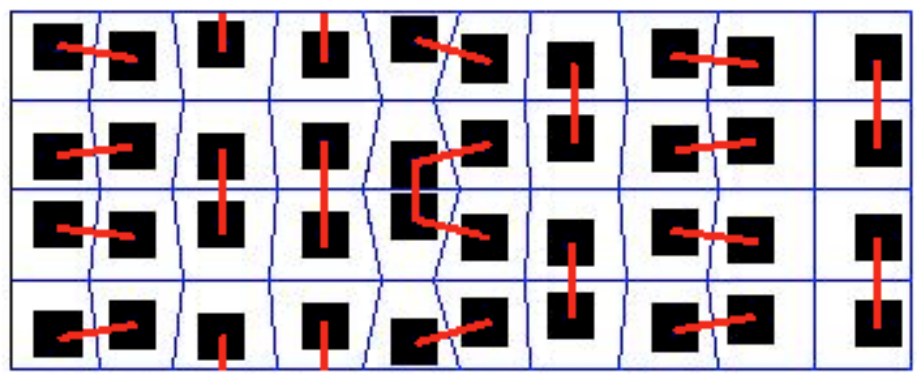

Figure 5. Voronoi polygons and nearest neighbor distances for each rod for a sample studied domain

Four different characteristics parameters are used to characterize the microstructure of the generated porous media and to distinguish them. These microstructural properties are standard deviation of Voronoi areas, nearest neighbour distances, neighbour orientation and finally mean nearest neighbor distance. Table 1 shows the calculated values of the aforementioned statistical descriptors for 6 random porous media named as Case Random 1 (i.e., CR1) to Case Random 6 (i.e., CR6). As it can be seen from Table 1, SD Voronoi areas, nearest neighbour distance and mean nearest neighbour distance are zero for the periodic and isotropic structure with inline arrangement. However, they are all different from zero for random porous structures. The maximum standard deviation of Voronoi areas, nearest neighbor distances, neighbor orientation and mean nearest neighbor distance are 0.124 for CR3, 0.152 for CR5, 1.727 for CR6 and finally 0.831 for CR1, respectively. Based on the obtained results, there is no relation between values of microstructural properties of different random porous media.

\section{Thermal behaviour of the studied random porous media}


As it was mentioned before, the velocity, pressure and temperature distributions are obtained for 6 different random rod arrangement when $\mathrm{Re}_{\mathrm{H}}=100$ which characteristic length defined as the height of the REV. Figure 6 shows the streamlines for the considered two REVs which have periodic inline and random arrangements.

Table 1

Microstructural properties of Studied Random Geometries (CR)

\begin{tabular}{cccccccc}
\hline Characteristics Parameters & Periodic & CR1 & CR2 & CR3 & CR4 & CR5 & CR6 \\
\hline SD Voronoi Areas & 0 & 0.098 & 0.072 & 0.124 & 0.077 & 0.043 & 0.063 \\
SD Nearest Neighbor Distance & 0 & 0.107 & 0.121 & 0.127 & 0.131 & 0.152 & 0.101 \\
SD Nearest Neighbor Orientation & 0.642 & 1.546 & 1.706 & 1.649 & 1.710 & 1.539 & 1.727 \\
Mean Nearest Neighbor Distance & 1 & 0.831 & 0.795 & 0.791 & 0.731 & 0.770 & 0.780 \\
\hline
\end{tabular}

Figure 6 (a) shows that the streamlines in a periodic structure are almost horizontal for upper and lower channels of the rods. Moreover, symmetrical vortices appear between the left and right walls of solid rods. Figure 6 (b) shows the streamlines in CR1. As expected, streamlines are not regular and flow paths around the rods are different from each other. The vortices with different sizes occur between walls at different locations due to the random positions of the solid rods.

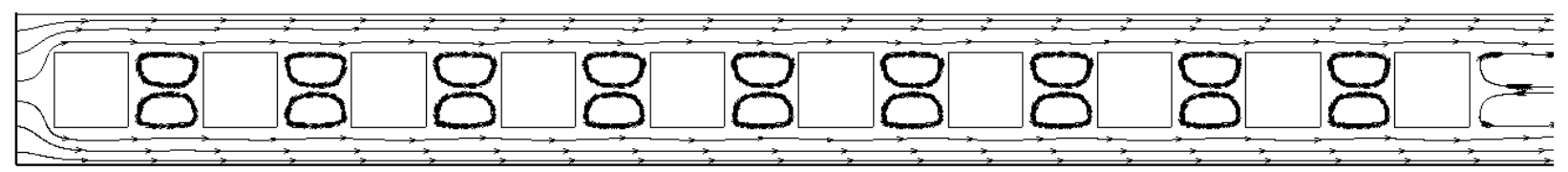

(a)

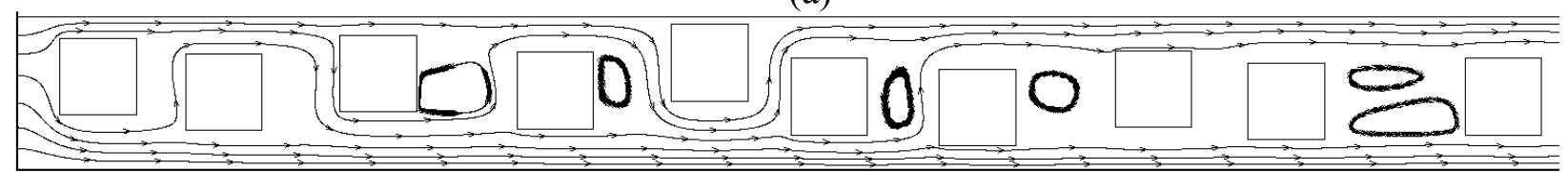

(b)

Figure 6. Streamlines in two considered REV for $\mathrm{Re}=100$

a) Periodic porous media with inline arrangement b) Randomly generated porous media (CR1)

Figure 7 shows the temperature distributions for 3 different random arrangements as CR1, CR2 and CR3. As it can be seen, the flow enters the 2D random porous medium from the left side and leaves the domain at the right side. Our observations showed that it is possible to classify rods into three groups as blocker, active and passive rods according to their effects on penetration of heat through the porous media. Each group is explained below.

Blocker rods: The blocker rods are those whose surfaces touch each other in the transverse direction of flow or they are very close to each other such that no or very small rate of fluid can flow between them. The blocker rods in Figure 7 are shown by black coloured rectangular boxes. If the gap between two rods behind the blocker rods is sufficiently large, fluid strikes the rods behind the blocker rods and then moves into the two channels behind the blocker rods perpendicular to the main flow direction. This causes the increase of heat transfer of the rods behind the blocker rods. Consequently, Nusselt number of the rods behind the block rods may increase. Figure 8 shows Nusselt number of the rods for CR1. As can be seen, the Nusselt number of rod behind the blocker rod increases due to the change of flow direction. 
Active rods: Active rods are the rods with a small distance between them such that fluid can flow in between. Fluid flowing towards an active rod strikes with the front wall of the rod and a part of the fluid flows through the gap between the rods and the rest of fluid moves toward the edges. The active rods in Figure 7 are shown by black coloured hexagon boxes. As expected, Nusselt number of the active rods should be higher since fluid collides with the front wall and flows over top and bottom horizontal walls of the rod as can be seen in Figure 8. It should be mentioned that overall Nusselt number of random porous media increases with an increase of the number of active rods.

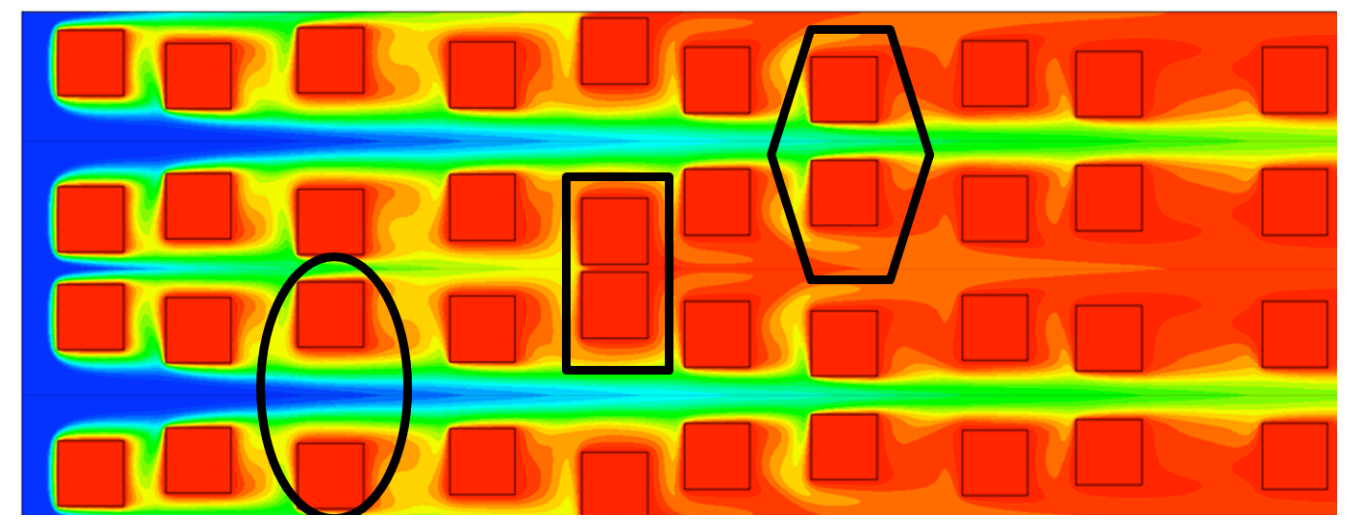

(a)

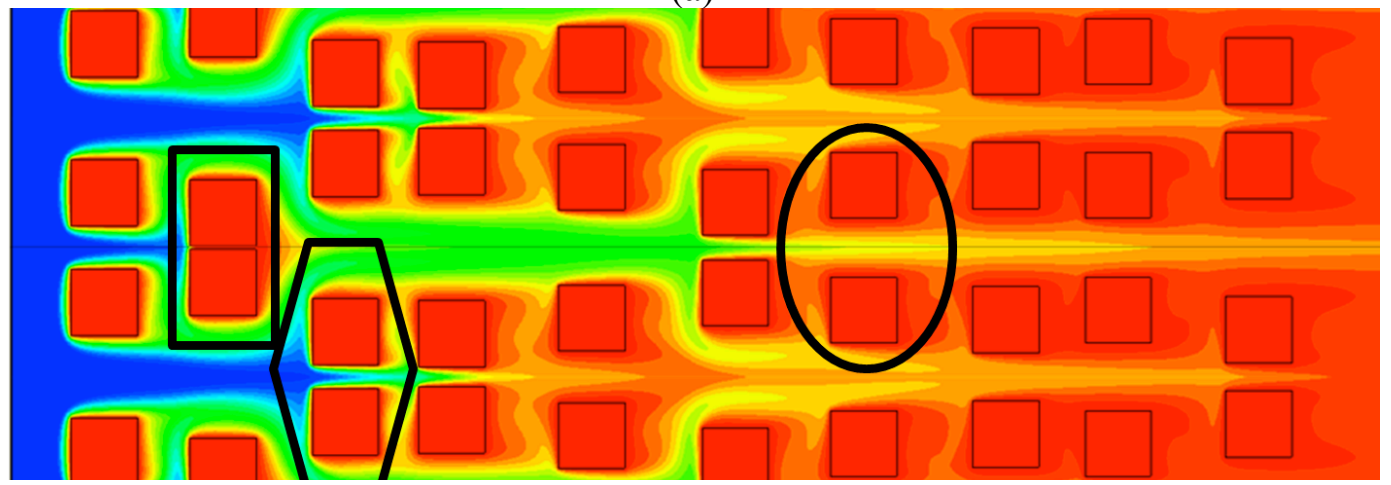

(b)

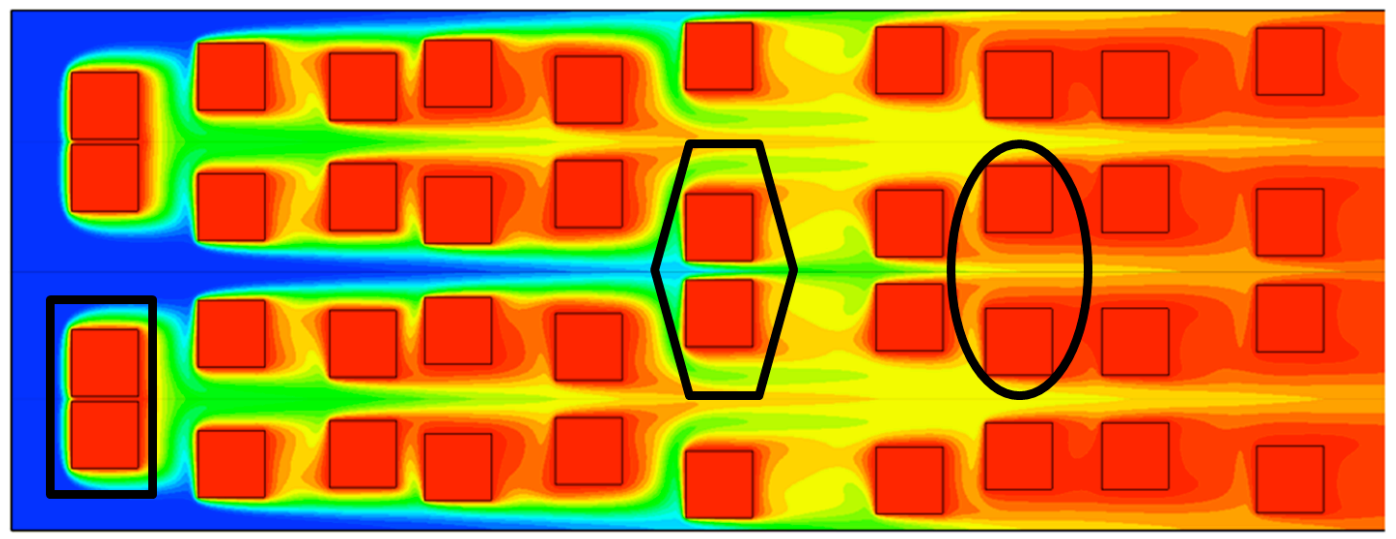

(c)

Temperature: 300.5301 .5302 .5303 .5304 .5305 .5306 .5307 .5308 .5309 .5

Figure 7. Temperature distribution and active rods (in a hexagon), passive rods (in an ellipse), blocker rods (in a rectangle) in random $2 \mathrm{D}$ porous media, a) CR1 b) CR2 c) CR3. 


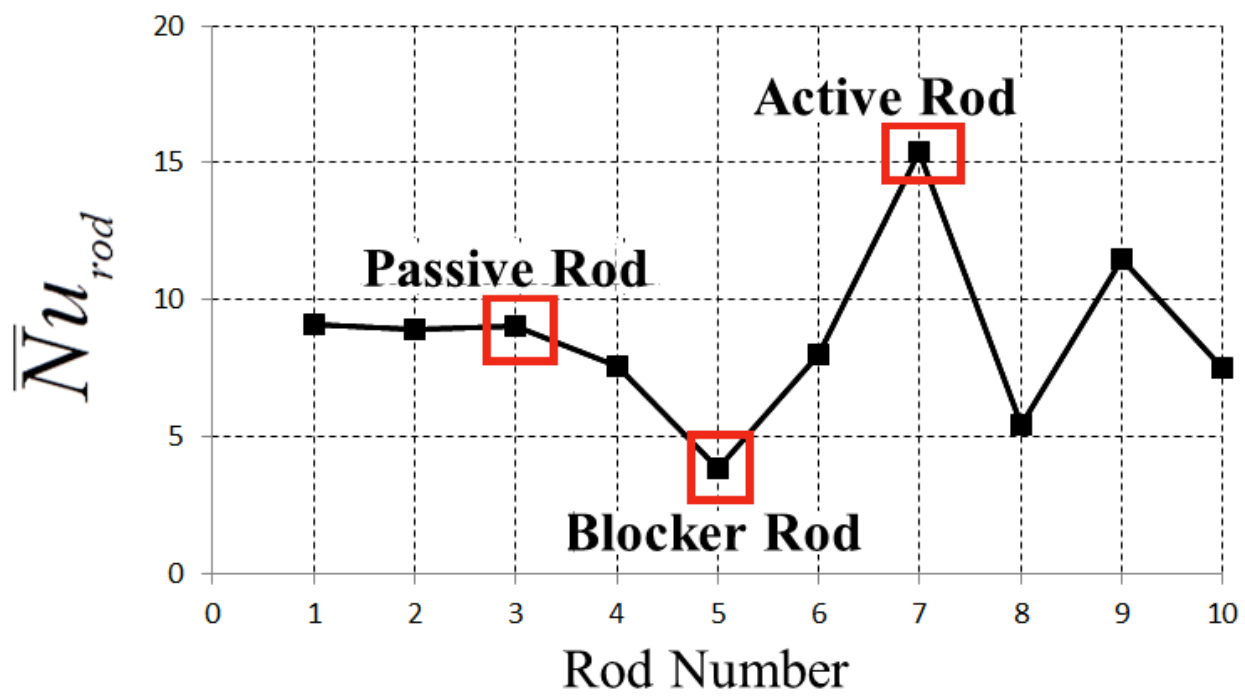

Figure 8. The effect of active, passive and blocker rods on average Nusselt number for CR1

Passive rods: Passive rods are the rods which are almost aligned behind a rod and they do not change the flow direction, effectively. The Nusselt number of a passive rod is considerably smaller than that of an active rod and this reduces the overall Nusselt number of the random porous media. Passive rods are shown by black colour ellipses in Figure 7. The comparison between Nusselt number of passive and active rods can be seen in Figure 8.

The average Nusselt numbers of each rod for 6 different porous media are shown in Figure 9 . Moreover the average Nusselt number of each rod of a periodic porous media with inline arrangement is shown in the same figure as a continuous line. As seen, the average Nusselt number of most rods in random porous media is greater than periodic inline arrangement. The Nusselt numbers of some rods in random porous media are around inline arrangement of rods.

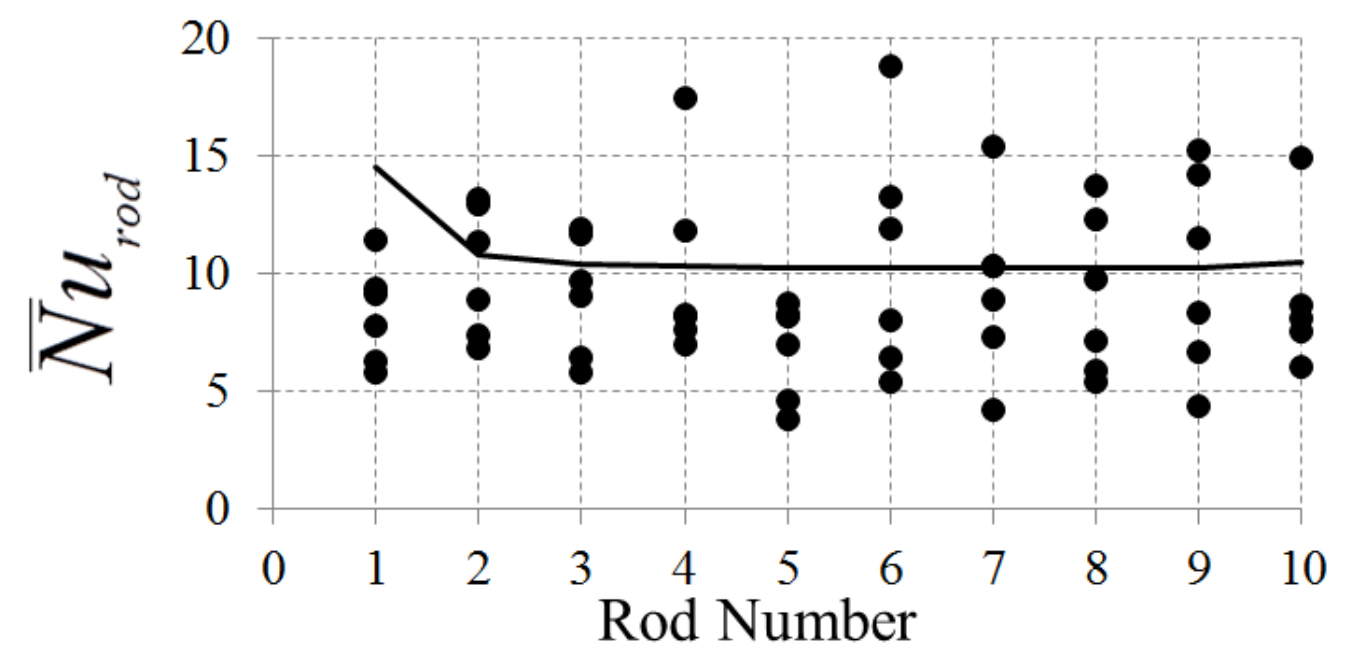

Figure 9. The average Nusselt number of each rod for the studied random porous media compared to the average Nusselts number for the inline arrangement of rods.

The overall Nusselt number of generated random porous media and the periodic one are compared in Figure 10. The overall Nusselt number of random porous media is less than the periodic inline 
arrangement for the most cases. The rate of decrease of overall Nusselt number is different for different random porous media.

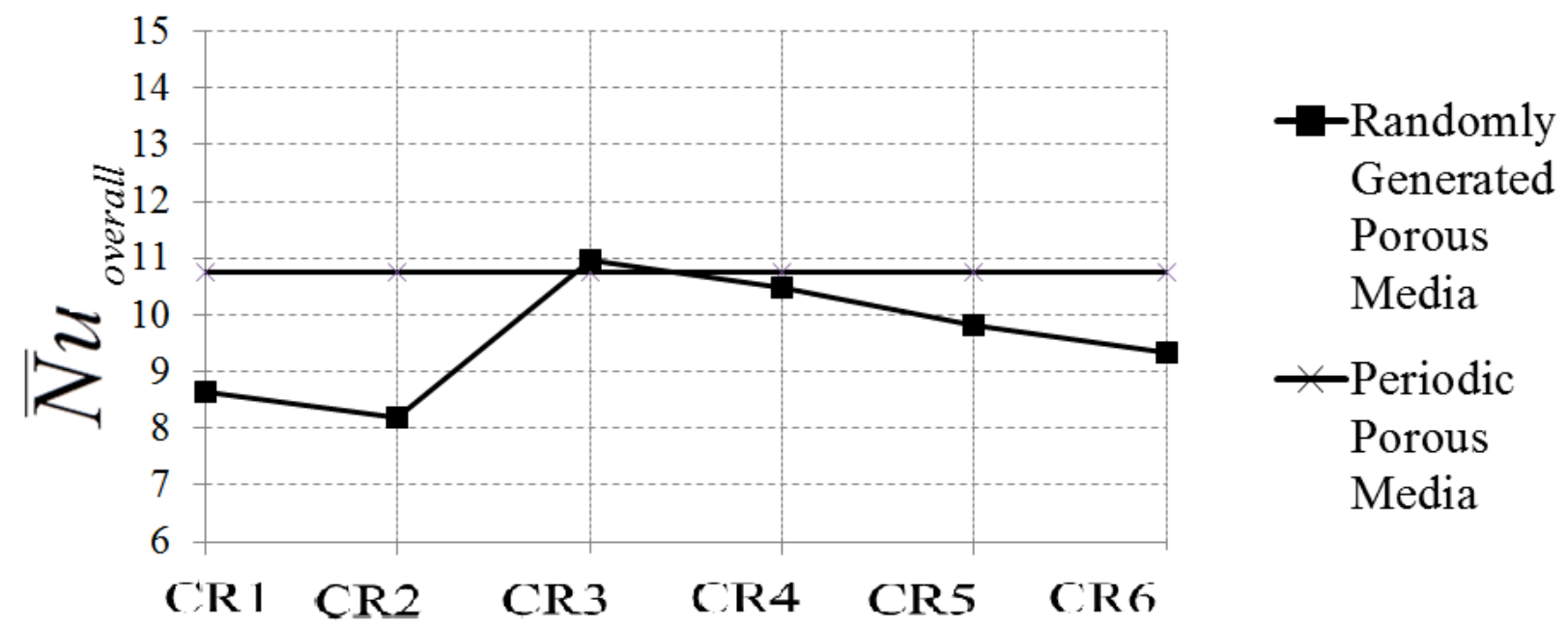

Figure 10. The overall Nusselt number for periodic inline arrangement and random structures

\section{CONCLUSION}

In this study 6 different random porous media involving 10 square rods are generated. The continuity, momentum and energy equations are solved for a row of rods representing the entire porous medium. The microstructure properties of each random porous medium which are standard deviations of Voronoi area, nearest neighbor distance and neighbor orientation and mean nearest neighbor distance are obtained to specify the generated domain. Nusselt numbers for each rod of the studied domain are determined based on the Voronoi area. The rods are classified into three groups as blocker, active and passive rods according to their effects on heat and fluid flow. There are rods in random porous media whose Nusselt number is greater than the inline periodic arrangement. However, the overall Nusselt number of random porous media is generally less than the one for the periodic one composed of an inline arrangement of rods.

For future study, the relation between the overall and/or average Nusselt numbers of random rods and microstructure properties of random porous media should be examined. Criteria for classification of rods into blocker, active and passive types of rods can be found and then based on number of these rods a relation for prediction of overall and/or average Nusselt numbers according to geometrical parameters may be found.

\section{REFERENCES}

Bowyer A. [1981], Computing Dirichlet Tessellations, The Computer Journal,Vol.24, No.2, pp.162166

Chen, X., Papathanasiouming T.D. [2007], Micro-Scale Modeling of Axial Flow through Unidirectional Disordered Fiber Arrays, Compos Sci Technol, Vol. 67, pp. 1286-1293

Chen, X., Papathanasiouming T.D. [2008] The Transverse Permeability of Disordered Fiber Arrays:

A Statistical Correlation in terms of the Mean Nearest Interfiber Spacing, Transp Porous Med, Vol. 
71, pp. 233-251.

Gamrat, G., Favre-Marinet, M. and Le Person, S. [2008], Numerical Study of Heat Transfer over banks of rods in small Reynolds number cross-flow, Int. J. Heat Mass Transfer, Vol. 51, pp. 853-864.

Guo X., Dai R. [2010], Numerical Simulation of Flow and Heat Transfer in a Random Packed Bed, Particuology, Vol. 8, pp. 293-299

Lopez Penha, D.J., Stolz, S., Kuerten, J.G.M., Nordlund, M., Kuczaj, A.K. and Geurts, B.J. [2012], Fully-Developed Conjugate Heat Transfer in Porous Media with Uniform Heating, Int. J. Heat Fluid Flow, Vol. 38, pp. 94-106

Nabovati, A., Sousa A.C.M. [2007],Fluid Flow Simulation in Random Porous Media at Pore Level Using the Lattice Boltzmann Method, J Eng Sci Technol Vol.2, No.3 pp. 226-237.

Nakayama, A., Kuwahara, F., Umemoto, T. and Hayashi, T. [ 2002], Heat and Fluid Flow within an Anisotropic Porous Medium, J. Heat Transfer, Vol. 124, pp. 746-753

Rahimian M. H., Pourshaghaghy A. [2002], Direct Simulation of Forced Convetion Flow in a Parallel Plate Channel Filled with Porous Media, Int Commun Heat Mass, Vol.29, No.6, pp. 867-878

Yazdchi, K., Srivastava, S. and Luding, S. [2012], Micro-Macro Relations for Flow through Random Arrays of Cylinders, Compos Part A, Vol. 43, pp. 2007-2020. 